\title{
Robotic measurement and control for chiropractic research
}

\author{
P. Goldsmith¹, S. Wynd ${ }^{1}$ and G. Kawchuk ${ }^{2}$ \\ ${ }^{1}$ University of Calgary, 2500 University Drive NW, Calgary, Alberta, Canada T2N 1N4 \\ ${ }^{2}$ University of Alberta, 114 St-89 Ave, Edmonton, Alberta, Canada T6G 2E1
}

doi:10.1533/abbi.2004.0042

\begin{abstract}
The precision and programmability of robotic manipulators makes them suitable for biomechanics research, particularly when an experimental procedure must be accurately repeated multiple times. This paper describes a robotic system used to investigate biomechanical mechanisms of stroke in humans. A parallel robot manipulator is used to reproduce chiropractic manipulations on animal subjects using a 3-D vision system. An algorithm for calibrating the system is proposed and tested on the robot. An iterative learning control scheme is then introduced to improve positional accuracy. Experimental results demonstrate that the calibration procedure and learning scheme are both effective.
\end{abstract}

Key words: Robotics, biomedical robots, chiropractic, robot calibration, vision-based motion control.

\section{INTRODUCTION}

The biomechanical mechanisms of stroke are not well understood. For example, it is not known if chiropractic manipulation can cause or exacerbate arterial lesions that result in stroke. This paper describes a robotic system being used to investigate such mechanisms.

This application is similar to that described in Goldsmith (1998) where a robotic system was proposed for testing sport shoes. The purpose of that system is to simulate human running motions in a repeatable way so that shoe performance can be objectively measured. In the present application, a robot simulates neck manipulations performed by a chiropractor, allowing repeatable experiments on the biomechanical mechanisms of stroke.

To accurately reproduce these motions, an effective calibration procedure is needed. This paper proposes an automatic calibration algorithm for the system that uses the minimum number of robot movements. The positional and rotational accuracy of the calibrated system is further improved by iteratively correcting robot motion commands using errors measured by the vision system.

\footnotetext{
Corresponding Author:

P. Goldsmith

Department of Mechanical and Manufacturing Engineering

University of Calgary, 2500 University Drive NW

Calgary, Alberta, Canada T2N 1N4

Email: peter.goldsmith@ucalgary.ca
}

\section{BIOMECHANICAL MECHANISMS OF STROKE}

Chiropractors routinely use cervical spinal manipulation (cSMT) for the treatment of musculoskeletal complaints of the head and neck. Although clinical studies have demonstrated some therapeutic benefits of cSMT (Whittingham and Nilsson 2001), serious complications may be associated with this procedure. Numerous case reports have cited chiropractic manipulation as a possible cause of cerebrovascular accidents, such as stroke (Parenti et al. 1999). Retrospective studies have also implicated cSMT as a cause of vertebral artery dissection (Haldeman et al. 2002). However, these studies do not establish a cause and effect relationship between chiropractic manipulation and stroke.

One hypothesis is that the biomechanical forces involved in chiropractic manipulation are sufficient to create a new injury either catastrophically or through cumulative insult to the vessel (Johnson et al. 2000). Investigators suspect that the anatomical configuration of the vertebral artery makes it vulnerable to injury. As the vertebral artery exits from the vertebral foramina of the first cervical vertebra and passes posteriorly through the foramen magnum, it creates a distinct "loop" (Thiel 1991). Due to its unique configuration at this level, investigators have hypothesized that chiropractic manipulation causes sufficient stresses and strains to kink (Pauliukas et al. 1989), stretch (Bladin and Merory 1975), or tear (Terrett 1987) the vessel wall causing thrombus formation (Frisoni and Anzola 1991) and ultimately vessel occlusion (Parkin et al. 1978).

A second hypothesis is that congenital malformations or pre-existing lesions make the vertebral artery 
susceptible to injury regardless of how trivial the applied forces are. Damaged or congenitally weakened vertebral arteries may remain subclinical until cumulative damage permits catastrophic failure to occur. Given that the reported symptoms associated with damage to the vertebral artery include headache, neck pain, localized stiffness, and nausea (Saeed et al. 2000), and given current public perception of chiropractor's scope of practice, these patients may present to a chiropractor for symptomatic relief. It is conceivable that symptoms felt by patients prior to their stroke are an indication of an underlying subclinical pathological lesion and that cSMT exacerbates a pre-existing injury in the vertebral artery.

The mechanisms of vertebral artery injury are poorly understood, while the resulting clinical effects of its injury have been well documented (Saeed et al. 2000). Precipitating events include major trauma, sports activities, and rotary head movement associated with chiropractic manipulation, yoga, and sitting in a hairdresser's chair (Haldeman et al. 1999). In some cases, spontaneous dissection has occurred in the absence of a precipitating event, suggesting that a pre-existing artery defect as a result of a congenital connective tissue disorder or vessel wall weakness preceded the injury (Haldeman et al. 1999). Due to the correlation between activity and onset of injury, investigators hypothesize that mechanical forces acting on the cervical spine during spinal manipulation can cause new injury to the vertebral artery (Panjabi et al. 1998). Since "spontaneous" injuries have been reported (von Pein et al. 2002), it may be that injuries are exacerbated in vessels with pre-existing pathological lesions as a result of the cumulative effects of cervical manipulation.

As a healthcare provider that promotes the safety of its treatment modalities, the chiropractic profession must establish safety guidelines and investigate all treatments that may pose a threat to public safety. The scope of this study is to establish possible links between pre-existing vertebral artery lesions and chiropractic manipulation of the cervical spine with a testing system capable of reproducing cervical spine movement in vivo (i.e. in a live subject).

\section{EXPERIMENTAL DESIGN}

Examining the hypothesis that chiropractors can exacerbate pre-existing lesions requires (1) a model of a pre-existing lesion, and (2) a method of applying cSMT repeatedly to this animal model. To date, we have developed an animal model of a pre-existing pathological lesion (Kawchuk et al. 2004). The development of a method of applying cSMT is the focus of this paper.

Complex motions of the type found in daily living are not easily studied ex vivo. We are developing a process to record spinal motion in vivo during a chiropractic cSMT and then apply those same motions in vivo by a robot. Unlike the chiropractor, the robot is capable of reproducing the same motion repeatedly, giving highly repeatable testing conditions.

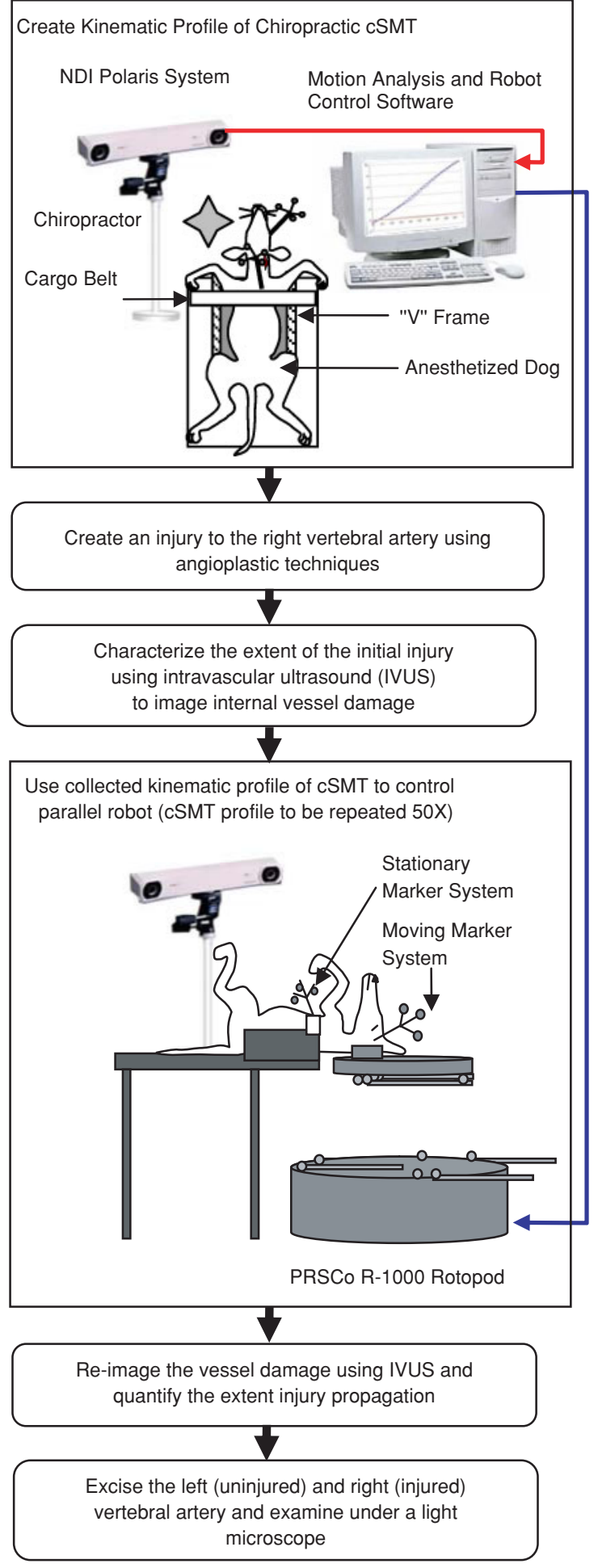

Figure 1 Schematic representation of the overall experimental protocol for chiropractic manipulation simulation.

The experimental design for this process is outlined schematically in Figure 1. A three-year-old $\operatorname{dog}$ is anesthetized and bone markers implanted for tracking the motion of the dog's cervical spine during chiropractic manipulation. One marker is implanted on the chest (stationary marker), while a second marker is implanted 


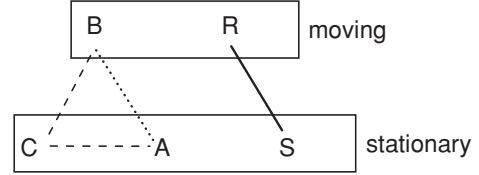

Figure 2 Coordinate frames.

on the skull (moving marker). Each marker is a triad of balls whose position and orientation is measured at $60 \mathrm{~Hz}$ by an NDI Polaris camera.

After the marker systems are implanted, an American Veterinary Chiropractic Association-certified practitioner will perform five rotary cervical spine manipulations applied to the $\mathrm{C} 2 / \mathrm{C} 3$ region, with a 1 min interval between manipulations. Forces applied to the spine during the manipulation will be within the therapeutic range for a canine procedure.

Following collection and analysis of the kinematic profiles of the cSMT, an angiographic balloon is inserted in the right vertebral artery to the level of the second or third cervical vertebra. The balloon is over-inflated, and translated within the vessel to create an injury. The balloon is then removed, and an intravascular ultrasound is inserted to image the initial damage to the artery. The left vertebral artery is not injured and acts as an internal control.

The dog is then relocated to the vicinity of the robot (the R-1000 Rotopod, manufactured by Parallel Robotic Systems Corporation, Hampton, NH). Its head is rigidly fixed to the robot platform and calibration of the robot is then performed. The robot then executes the recorded motions of the cSMT 50 times. The dog is then removed from the robot, and the IVUS is reinserted into the right vertebral artery to examine changes to the injury. Changes in injury volume, length and diameter are noted. The left and right vertebral arteries are then removed postmortem and compared using light microscopy.

If the injury worsens, this will support our hypothesis that chiropractic cSMT can exacerbate a pre-existing lesion.

\section{CALIBRATION ALGORITHM}

The robot must reproduce the cSMT motions of the chiropractor recorded by the camera. This requires a calibration of the robot to determine the location of the robot frames and bone markers relative to the camera. Here we propose an algorithm to find these locations automatically with a minimum of robot motion commands.

\section{Problem statement}

As depicted in Figure 2, the coordinate frames are labeled as:

C, camera frame (stationary)

A, chest bone marker (stationary)

$S$, robot base frame (stationary)

$\mathrm{R}$, robot platform frame (moving)

$\mathrm{B}$, skull bone marker (moves rigidly with $\mathrm{R}$ )
The relative locations of frames are represented by homogeneous transformation matrices. For example, the location of A relative to $\mathrm{C}$ is denoted $H_{\mathrm{C}}^{\mathrm{A}}$. The dashed lines in Figure 2 indicate measured relations, the dotted line indicates the relation we wish to control, the solid line indicates the relation we can command to the robot, and absent lines indicate unknown relations (prior to calibration).

To move $H_{\mathrm{A}}^{\mathrm{B}}$ to a desired value $H_{\mathrm{A}}^{\mathrm{B}_{\mathrm{d}}}$ (recorded from the cSMT), we move the robot to

$$
H_{\mathrm{S}}^{\mathrm{R}}=H_{\mathrm{S}}^{\mathrm{A}} H_{\mathrm{A}}^{\mathrm{B}_{\mathrm{d}}} H_{\mathrm{B}}^{\mathrm{R}} .
$$

However, $H_{\mathrm{S}}^{\mathrm{A}}$ and $H_{\mathrm{B}}^{\mathrm{R}}$ are unknown, since the camera cannot see frames $\mathrm{S}$ or $\mathrm{R}$. The calibration problem is to deduce these locations from camera measurements and known robot motions.

To find $H_{\mathrm{S}}^{\mathrm{A}}$ and $H_{\mathrm{B}}^{\mathrm{R}}$, it is sufficient to find $H_{\mathrm{C}}^{\mathrm{S}}$, since

$$
H_{\mathrm{S}}^{\mathrm{A}}=\left(H_{\mathrm{C}}^{\mathrm{S}}\right)^{-1} H_{\mathrm{C}}^{\mathrm{A}}
$$

and (for a known robot position $H_{\mathrm{S}}^{\mathrm{R}}$ )

$$
H_{\mathrm{B}}^{\mathrm{R}}=\left(H_{\mathrm{C}}^{\mathrm{B}}\right)^{-1} H_{\mathrm{C}}^{\mathrm{S}} H_{\mathrm{S}}^{\mathrm{R}}
$$

Thus, the calibration problem reduces to finding $H_{\mathrm{C}}^{\mathrm{S}}$ in a minimum of robot moves.

\section{A solution}

Since a marker location yields six independent data (three position coordinates and three orientation coordinates), one might conjecture that it is possible to deduce $H_{\mathrm{C}}^{\mathrm{S}}$ by measuring $H_{\mathrm{C}}^{\mathrm{B}}$ before and after a single known move. This conjecture is incorrect because two components of $H_{\mathrm{C}}^{\mathrm{B}}$ are known a priori from $H_{\mathrm{S}}^{\mathrm{R}}$ : the magnitude of rotation and the magnitude of translation normal to the plane of rotation. Therefore, only four new components can be obtained from a single move, implying that two moves are required to obtain all six components of $H_{\mathrm{C}}^{\mathrm{S}}$.

We will use rotations to find $H_{\mathrm{C}}^{S}$ since a pure translation yields only two coordinates (the azimuth and elevation of the translation axis). We use the notation

$$
H_{b}^{a}=\left[\begin{array}{cc}
R_{b}^{a} & d_{b}^{a} \\
0 & 1
\end{array}\right]=\left[\begin{array}{cccc}
x_{b}^{a} & y_{b}^{a} & z_{b}^{a} & d_{b}^{a} \\
0 & 0 & 0 & 1
\end{array}\right] .
$$

The calibration procedures are:

(1) Command the robot to move to a suitable location $H_{\mathrm{S}}^{\mathrm{R} 0}$ for mounting the test subject.

(2) Fix chest (A) to table and skull (B) to robot.

(3) Record $H_{\mathrm{C}}^{\mathrm{B} 0}=$ initial location of marker $\mathrm{B}$.

(4) Command a pure roll of $\alpha_{x}$ about $x^{\mathrm{S}}$ axis.

(5) Record $H_{\mathrm{C}}^{\mathrm{B} 1}=$ new location of marker $\mathrm{B}$.

(6) Command a pure roll of $\alpha_{y}$ about $y^{\mathrm{S}}$ axis, measured relative to the initial location.

(7) Record $H_{\mathrm{C}}^{\mathrm{B} 2}=$ new location of marker $\mathrm{B}$.

The first two columns of $H_{\mathrm{C}}^{\mathrm{S}}$ are given by the roll axis $x_{\mathrm{C}}^{\mathrm{S}}$ and pitch axis $y_{\mathrm{C}}^{\mathrm{S}}$ (in camera coordinates). The third column is $z_{\mathrm{C}}^{\mathrm{S}}=x_{\mathrm{C}}^{\mathrm{S}} \times y_{\mathrm{C}}^{\mathrm{S}}$. Then we find a point $p^{x}$ on $x^{\mathrm{S}}$ 
and a point $p^{y}$ on $y^{\mathrm{S}}$, and thus the origin $d_{\mathrm{C}}^{\mathrm{S}}$ of frame $\mathrm{S}$ (column 4 of $H_{\mathrm{C}}^{\mathrm{S}}$ ).

The vector $x_{\mathrm{C}}^{\mathrm{S}}$ is given by

$$
x_{\mathrm{C}}^{\mathrm{S}}=\frac{1}{2 \sin \alpha_{x}}\left(\begin{array}{l}
X_{32}-X_{23} \\
X_{13}-X_{31} \\
X_{21}-X_{12}
\end{array}\right),
$$

where $X$ represents a roll of $\alpha_{x}$ about $x^{\mathrm{S}}$ and thus satisfies

$$
R_{\mathrm{C}}^{\mathrm{B} 1}=X R_{\mathrm{C}}^{\mathrm{B} 0} .
$$

Similarly, $y_{\mathrm{C}}^{\mathrm{S}}$ is found from $Y=R_{\mathrm{C}}^{\mathrm{B} 2}\left(R_{\mathrm{C}}^{\mathrm{B} 0}\right)^{-1}$.

The steps in the calibration algorithm are:

1. Find $x_{\mathrm{C}}^{\mathrm{S}}$ from (5) and (6), and normalize it as $x_{\mathrm{C}}^{\mathrm{S}}=$ $x_{\mathrm{C}}^{\mathrm{S}} /\left\|x_{\mathrm{C}}^{\mathrm{S}}\right\|$.

2. Find $y_{\mathrm{C}}^{\mathrm{S}}$ similarly from $Y$.

3. Find $z_{\mathrm{C}}^{\mathrm{S}}=x_{\mathrm{C}}^{\mathrm{S}} \times y_{\mathrm{C}}^{\mathrm{S}}$ and normalize it.

4. Find $y_{\mathrm{C}}^{\mathrm{S}}=z_{\mathrm{C}}^{\mathrm{S}} \times x_{\mathrm{C}}^{\mathrm{S}}$.

5. $p_{\mathrm{C}}^{x}=0.5\left[d_{\mathrm{C}}^{\mathrm{B} 0}+d_{\mathrm{C}}^{\mathrm{B} 1}+\cot \left(0.5 \alpha_{x}\right)\left(x_{\mathrm{C}}^{\mathrm{S}} \times\left(d_{\mathrm{C}}^{\mathrm{B} 1}-d_{\mathrm{C}}^{\mathrm{B} 0}\right)\right]\right.$.

6. $p_{\mathrm{C}}^{y}=0.5\left[d_{\mathrm{C}}^{\mathrm{B} 0}+d_{\mathrm{C}}^{\mathrm{B} 2}+\cot \left(0.5 \alpha_{y}\right)\left(y_{\mathrm{C}}^{\mathrm{S}} \times\left(d_{\mathrm{C}}^{\mathrm{B} 2}-d_{\mathrm{C}}^{\mathrm{B} 0}\right)\right]\right.$.

7. $d_{\mathrm{C}}^{\mathrm{S}}=p_{\mathrm{C}}^{x}+\left[\left(p_{\mathrm{C}}^{y}-p_{\mathrm{C}}^{x}\right)^{T} x_{\mathrm{C}}^{\mathrm{S}}\right] x_{\mathrm{C}}^{\mathrm{S}}$.

8. $H_{\mathrm{C}}^{\mathrm{S}}=\left[\begin{array}{cccc}x_{\mathrm{C}}^{\mathrm{S}} & y_{\mathrm{C}}^{\mathrm{S}} & z_{\mathrm{C}}^{\mathrm{S}} & d_{\mathrm{C}}^{\mathrm{S}} \\ 0 & 0 & 0 & 1\end{array}\right]$.

Due to measurement error, the $y_{\mathrm{C}}^{\mathrm{S}}$ found in step 2 will not be exactly perpendicular to $x_{C}^{S}$. Steps 3 and 4 ensure that all three axes are perpendicular. Step 5 is obtained from the triangle with vertices $d^{\mathrm{B} 0}, p^{x}, d^{\mathrm{B} 1}$ and interior angle at $p^{x}$ of $\alpha_{x}$. Step 6 is similarly obtained from the triangle $d^{\mathrm{B} 0}, p^{y}, d^{\mathrm{B} 2}$, whose interior angle at $p^{y}$ is $\alpha_{y}$.

Step 7 gives the origin of Frame $S$ by projecting $p^{x}$ and $p^{y}$ onto its axes, and alternatively, $d_{\mathrm{C}}^{\mathrm{S}}=p_{\mathrm{C}}^{y}+\left[\left(p_{\mathrm{C}}^{x}-\right.\right.$ $\left.\left.p_{\mathrm{C}}^{y}\right)^{T} y_{\mathrm{C}}^{s}\right] y_{\mathrm{C}}^{\mathrm{s}}$. This reflects the redundancy in the data (i.e. eight data to find six components).

\section{CALIBRATION ACCURACY}

Following calibration, the robot was commanded to move to the four locations given in Table 1 , and the actual robot locations were measured based on camera data for marker B. These measured locations appear in the table, along with the size of the translational and rotational errors. The rotational error is computed as

$$
e_{\text {rot }}=\cos ^{-1}\left(\left(0.5 \operatorname{Tr}\left(R_{\mathrm{S}}^{\mathrm{R}}\right)\left(R_{\mathrm{S}}^{\mathrm{R}}\right)_{m}^{T}\right)-0.5\right)
$$

where $R_{\mathrm{S}}^{\mathrm{R}}$ is the commanded rotation matrix and $\left(R_{\mathrm{S}}^{\mathrm{R}}\right)_{m}$ is the measured rotation.

Locations $\mathrm{A}$ and $\mathrm{B}$ represent a pure translation and a pure rotation, respectively. Location $\mathrm{C}$ was a point used in the calibration, while location $D$ represents a large arbitrary motion. As expected, point $\mathrm{C}$ yields the smallest error, which indicates the robot's ability to return to a known

\begin{tabular}{|c|c|c|c|c|}
\hline & & $\begin{array}{l}\text { Robot } \\
\text { command }\end{array}$ & $\begin{array}{l}\text { Measured } \\
\text { movement }\end{array}$ & Error \\
\hline \multirow{6}{*}{ A } & Roll & 0 & -0.0828 & \multirow{3}{*}{$0.25^{\circ}$} \\
\hline & Pitch & 0 & -0.0911 & \\
\hline & Yaw & 0 & 0.0538 & \\
\hline & $\mathrm{X}$ & 10 & 10.1563 & \multirow{3}{*}{$0.61 \mathrm{~mm}$} \\
\hline & Y & 5 & 4.4335 & \\
\hline & Z & 10 & 9.8185 & \\
\hline \multirow[t]{6}{*}{ B } & Roll & 5 & 4.9767 & \multirow{4}{*}{$0.60^{\circ}$} \\
\hline & Pitch & 7 & 7.0906 & \\
\hline & Yaw & 9 & 9.0027 & \\
\hline & $\mathrm{X}$ & 0 & -0.0727 & \\
\hline & $\mathrm{Y}$ & 0 & -0.1101 & \multirow[t]{2}{*}{$1.1 \mathrm{~mm}$} \\
\hline & Z & 0 & 0.3021 & \\
\hline \multirow[t]{6}{*}{ C } & Roll & 0 & 0.0325 & \multirow{4}{*}{$0.12^{\circ}$} \\
\hline & Pitch & 10 & 10.1167 & \\
\hline & Yaw & 0 & 0.0033 & \\
\hline & $\mathrm{X}$ & 0 & -0.6471 & \\
\hline & Y & 0 & 0.1569 & \multirow[t]{2}{*}{$0.71 \mathrm{~mm}$} \\
\hline & Z & 0 & 0.1641 & \\
\hline \multirow[t]{6}{*}{$\mathrm{D}$} & Roll & 9.52 & 8.96 & \multirow{4}{*}{$0.91^{\circ}$} \\
\hline & Pitch & -10.96 & -10.24 & \\
\hline & Yaw & 9.62 & 9.70 & \\
\hline & $\mathrm{X}$ & 30.00 & 29.41 & \\
\hline & $\mathrm{Y}$ & 40.00 & 39.12 & \multirow{2}{*}{$2.8 \mathrm{~mm}$} \\
\hline & Z & 15.00 & 16.62 & \\
\hline
\end{tabular}
point (i.e. the robot's repeatability).
Table 1 Accuracy following calibration

\section{ITERATIVE LEARNING ALGORITHM}

In (1), $H_{\mathrm{S}}^{\mathrm{A}}$ may be expanded by the identity

$$
\begin{aligned}
H_{\mathrm{S}}^{\mathrm{A}} & =H_{\mathrm{S}}^{\mathrm{R} 0} H_{\mathrm{R} 0}^{\mathrm{B} 0} H_{\mathrm{B} 0}^{\mathrm{C}} H_{\mathrm{C}}^{\mathrm{A}} \\
& =H_{\mathrm{S}}^{\mathrm{R} 0} H_{\mathrm{R}}^{\mathrm{B}} H_{\mathrm{B} 0}^{\mathrm{C}} H_{\mathrm{C}}^{\mathrm{A}},
\end{aligned}
$$

where the second equality follows from the fact that $H_{\mathrm{R}}^{\mathrm{B}}=$ $H_{\mathrm{R} 0}^{\mathrm{B} 0}$ is constant, since frames $\mathrm{B}$ and $\mathrm{R}$ are rigidly connected. Substituting (8) into (1) gives

$$
H_{\mathrm{S}}^{\mathrm{R}}=\left(H_{\mathrm{S}}^{\mathrm{R} 0}\right) H_{\mathrm{R}}^{\mathrm{B}}\left(H_{\mathrm{B} 0}^{\mathrm{C}}\right) H_{\mathrm{C}}^{\mathrm{A}} H_{E}^{\mathrm{B}_{d}} H_{\mathrm{B}}^{\mathrm{R}},
$$

where the bracketed terms show the dependence on the calibration point $\left(\mathrm{R}_{0}, \mathrm{~B}_{0}\right)$.

If $\mathrm{B}_{d}=\mathrm{B}_{0}$, (9) reduces to $H_{\mathrm{S}}^{\mathrm{R}}=H_{\mathrm{S}}^{\mathrm{R} 0}$, which implies $\mathrm{B}=\mathrm{B}_{0}=\mathrm{B}_{\mathrm{d}}$. This is true even if calibration error exists in $H_{\mathrm{R}}^{\mathrm{B}}$ or if the robot positioning system is inaccurate (as long as it is repeatable). In other words, we should expect zero error in the location of Marker B if its desired location is the calibration point. This was effectively confirmed in the previous section, where it was also shown that error increases with distance from the calibration point.

We can exploit these observations by writing (9) in terms of a different point $B_{1}$, for which the corresponding robot command $H_{\mathrm{S}}^{\mathrm{R} 1}$ is known. An identity analogous to (8), 


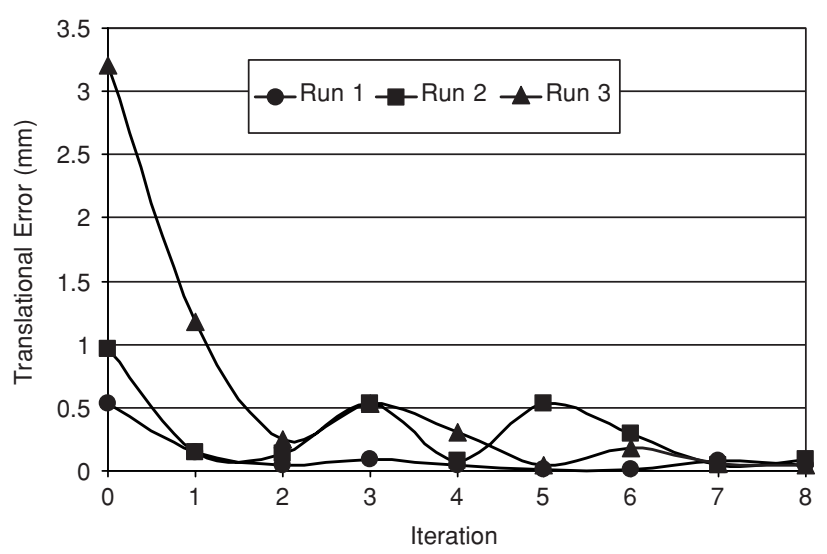

Figure 3 Translational errors versus iteration number.

substituted in (1), gives

$$
H_{\mathrm{S}}^{\mathrm{R}}=\left(H_{\mathrm{S}}^{\mathrm{R} 1}\right) H_{\mathrm{R}}^{\mathrm{B}}\left(H_{\mathrm{B} 1}^{\mathrm{C}}\right) H_{\mathrm{C}}^{\mathrm{A}} H_{E}^{\mathrm{B}_{d}} H_{\mathrm{B}}^{\mathrm{R}}
$$

As before, if $\mathrm{B}_{d}=\mathrm{B}_{1}$ in (10), then $\mathrm{B}=\mathrm{B}_{1}=\mathrm{B}_{d}$. So the control (10) can improve on (9). We can also expect that if $\mathrm{B}_{d}$ is close to $\mathrm{B}_{1}$, the error will be smaller that that produced by (9).

Since every move/measurement pair $\left(\mathrm{R}_{i}, \mathrm{~B}_{i}\right)$ gives a new "calibration point" in (10), and since the effectiveness of the calibration point generally improves with proximity to the target point $\mathrm{B}_{d}$, iterative application of (10) should result in convergence to the target. This iterative learning algorithm may be written as

$$
\left(H_{\mathrm{S}}^{\mathrm{R}}\right)_{i}=\left(H_{\mathrm{S}}^{\mathrm{R}}\right)_{i-1} H_{\mathrm{R}}^{\mathrm{B}}\left(H_{\mathrm{B}}^{\mathrm{C}}\right)_{i-1} H_{\mathrm{C}}^{\mathrm{A}} H_{\mathrm{A}}^{\mathrm{B}_{d}} H_{\mathrm{B}}^{\mathrm{R}}
$$

where $i$ is the iteration number. The fixed point of (11) is obtained by setting $\left(H_{\mathrm{S}}^{\mathrm{R}}\right)_{i}=\left(H_{\mathrm{S}}^{\mathrm{R}}\right)_{i-1}$, which gives the unique solution $H_{\mathrm{B}_{i-1}}^{\mathrm{B}_{d}}=I$, implying zero error $\left(\mathrm{B}_{i-1}=\right.$ $\left.\mathrm{B}_{d}\right)$.

\section{ITERATIVE LEARNING EXPERIMENT}

The iterative learning scheme (11) was applied to the robot at location $\mathrm{D}$, where the largest errors occurred. Three runs of the learning scheme were applied over two days, each run consisting of eight learning iterations. The resulting translational and rotational errors are plotted in Figures 3 and 4 as functions of the iteration number.

The zeroith iteration gives the robot's initial accuracy at point $\mathrm{D}$ before iterative learning is applied. This initial accuracy varies over runs 1-3 due to lack of repeatability. Run 1 occurred on the first day of the experiment, while runs 2 and 3 occurred on the second day.

Figures 3 and 4 show that the errors converged to nearly zero after eight learning iterations. The convergence is not monotonic, perhaps due to lack of repeatability from iteration to iteration. Following the experiments, an error was found in the code used to transform robot commands in matrix form to roll-pitch-yaw-xyz coordinates. Thus, Figures 3 and 4 demonstrate that the learning algorithm (11)

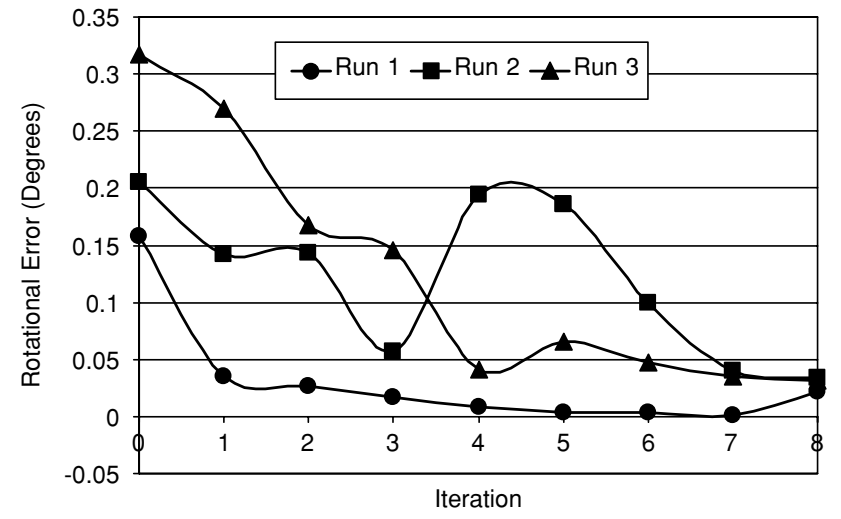

Figure 4 Rotational errors versus iteration number.

converges not only in the presence of expected calibration errors, but also in the presence of transformation errors. This typifies the nature of corrective algorithms. The errors in Figures 3 and 4 are calculated correctly based on camera data for marker B locations and they do not include the noted transformation error. The calibration results discussed earlier (see "Calibration accuracy" section) are similarly unaffected.

\section{CONCLUSIONS}

We have described a robotic system for investigating the biomechanical mechanisms of stroke. A calibration procedure for the robotic system was proposed that uses the minimum number of robot movements. This calibration yielded reasonable system accuracy relative to repeatability errors. The proposed iterative learning scheme further improved the accuracy of the system and converged in the face of calibration errors, repeatability errors, and a transformation error.

\section{REFERENCES}

Bladin PF, Merory J. 1975. Mechanisms in cerebral lesions in trauma to high cervical portion of the vertebral artery-rotation injury. Proc Aust Assoc Neurol, 12:35-41.

Frisoni GB, Anzola GP. 1991. Vertebrobasilar ischemia after neck motion. Stroke, 22:1452-1460.

Goldsmith P. 1998. Design of a robot for sport shoe testing. In Proceeding of the 5th International Conference on Control, Automation, Robotics, and Vision, Singapore, Dec. 9-11, pp. $105-110$.

Haldeman S, Kohlbeck F, McGregor M. 1999. Risk factors and precipitating neck movements causing vertebrobasilar artery dissection after cervical trauma and spinal manipulation. Spine, 24:785-794.

Haldeman S, Kohlbeck F, McGregor M. 2002. Unpredictability of cerebrovascular ischemia associated with cervical spine manipulation therapy. Spine, 27:49-55.

Johnson CP, How T, Scraggs M, West CR, Burns J. 2000. A biomechanical study of the human vertebral artery with implications for fatal arterial injury. Forensic Sci Int, 109:169-182. 
Kawchuk GN, Wynd S, Anderson T. 2004. Defining the effect of cervical manipulation on vertebral artery integrity: Establishment of an animal model. 7 Manipulative Physiol Ther 27:539-546.

Panjabi MM, Cholewicki J, Nibu K et al.. 1998. Simulation of whiplash trauma using whole cervical spine specimens. Spine, 23:17-24.

Parenti G, Orlandi G, Bianchi M et al.. 1999. Vertebral and carotid artery dissection following chiropractic cervical manipulation. Neurosurg Rev, 22:127-129.

Parkin PJ, Wallis WE, Wilson JL. 1978. Vertebral artery occlusion following manipulation of the neck. $N Z \mathrm{Med} \mathcal{F}$, 88:441-443.

Pauliukas PA, Matskevichus ZK, Barkauskas EM. 1989. Changes in the internal carotid artery in its loop-shaped kinking and their clinical significance. Khirurgiia (Mosk), Sep(9):47-51.
Saeed AB, Shuaib A, Al-Sulaiti G, Emery D. 2000. Vertebral artery dissection: Warning symptoms, clinical features and prognosis in 26 patients. Can $\mathcal{F}$ Neurol Sci, 27:292-296.

Terrett A. 1987 Vascular accidents from cervical spine manipulation: Report on 107 cases. 7 Aust Chiropractors Assoc., 17:15-23.

Thiel H. 1991. Gross Morphology and pathoanatomy of the vertebral arteries. 7 Manipulative Physiol Ther, 14:133141.

von Pein F, Valkkila M, Schwarz R et al.. 2002. Analysis of the COL3A1 gene in patients with spontaneous cervical artery dissections. 7 Neurol, 249:862-866.

Whittingham W, Nilsson N. 2001. Active range of motion in the cervical spine increases after spinal manipulation (toggle recoil). 7 Manipulative Physiol Ther, 24:552-555. 

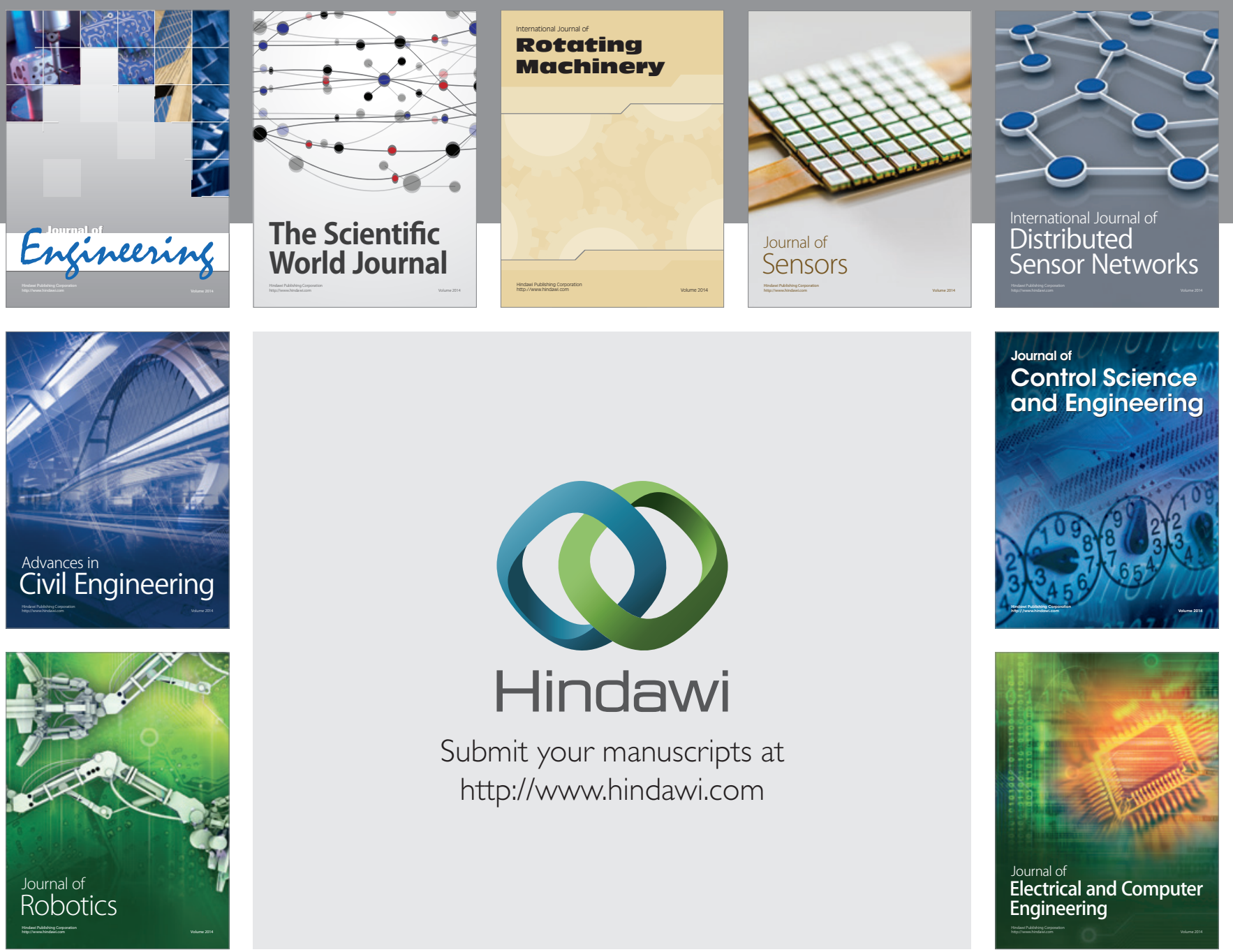

Submit your manuscripts at

http://www.hindawi.com
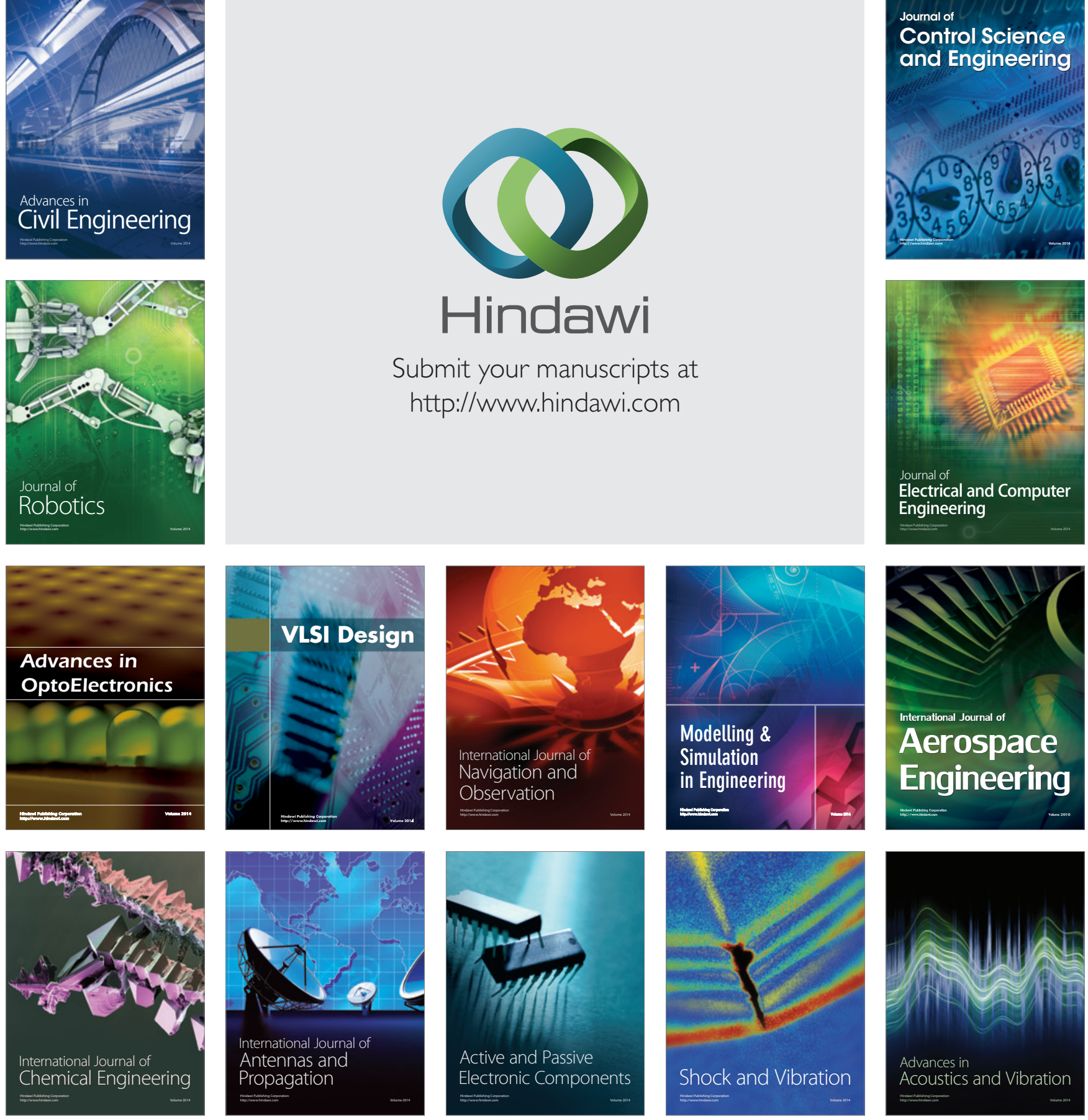